\title{
A Single Feed Circularly Polarized Planar Antenna Array
}

\author{
Rabindra Kumar, Neha Kumari, Pradeep Kumar Jain, Priyanka Mondal
}

\begin{abstract}
A design of a circularly polarized planar antenna array of elliptical patches is presented. The designed prototype has been verified experimentally. Elliptical patches produce circular polarization by using a single feed only. A corporate feed network with quarter-wave transformers are used for uniform excitation of all the array elements. Primary advantages of the presented antenna array are structural simplicity, good circular polarization characteristics and no side lobes. Here, the design example is given for $2 \times 2$ elliptical patches at $2.8 \mathrm{GHz}$. Similar procedure can be extended for more number of array elements at the desired frequency of operation to cater the need of communication systems where circular polarization is essential.
\end{abstract}

Keywords: Antenna array, axial ratio, circular polarization, elliptical patch, microstrip, single feed

\section{INTRODUCTION}

Circularly polarized waves radiate energy in the horizontal and vertical plane, as well as every plane in between. So, circular polarization (CP) is more resistant to signal degradation due to atmospheric conditions, such as rotation of signal polarization. Circularly polarized waves do not observe Faraday rotation due to Earth's magnetic field while passing through ionosphere. Also, Faraday rotation is less noticeable at higher frequency, e.g., $\mathrm{Ku}$-band. So, for Satellite communication at lower microwave frequencies circularly polarized antennas are very useful. Microstrip antennas [1] being low profile planar structure are very effective radiators. A single patch antenna can be made to radiate circular polarization if two orthogonal patch modes [2] are simultaneously excited with equal amplitude but in phase quadrature with sign determining the sense of rotation. Two types of feeding schemes can accomplish this task. The first type is a dual-orthogonal feed, which employs an external power divider network in the form of a quadrature hybrid or a T-junction power divider with a quarter-wavelength line.

Revised Manuscript Received on December 30, 2019.

* Correspondence Author

Rabindra kumar, Electronics and Communication Engg. Department, National Institute of Technology Patna (Bihar) India. E-mail; rk_nitp@nitp.ac.in.,

Neha kumari, Electronics and Communication Engg. Department,

National Institute of Technology Patna (Bihar) India. E-mail; neha.raj8276@gmail.com

Pradeep kumar jain, Electronics and Communication Engg. Department, National Institute of Technology Patna (Bihar) India. E-mail; pkjain@nitp.ac.in

Priyanka mondal, Electronics and Communication Engg. Department, National Institute of Technology Patna (Bihar) India. E-mail; pmondal@nitp.ac.in

(C) The Authors. Published by Blue Eyes Intelligence Engineering and Sciences Publication (BEIESP). This is an open access article under the CC BY-NC-ND license (http://creativecommons.org/licenses/by-nc-nd/4.0/)
Evidently, the design of feed network becomes complicated while designing an array antenna. The other is single feed and CP is obtained by slightly perturbing the microstrip patch at appropriate location with respect to the feed.In [3], it is shown that CP is obtained from single feed circular patch antenna tuned by passive metallic or shorting posts.

A class of CP antennas are derived from disk and square microstrip antennas by cutting slots in their interiors [4] or corners [5] off their perimeters. It eliminates the necessity of complex feed network as mentioned above but requires to cut the slots of appropriate size at the right position.

Another simple way to produce left handed or right handed circularly polarized radiation is to make a single feed circular patch elliptical in shape with small eccentricity [6], [7]. It creates two spatially orthogonal degenerate modes along the two axes of ellipse. These two modes are in phase quadrature if the feed line is chosen along the line made $45^{0}$ with respect to the axes. Surface currents, internal fields and radiated fields are obtained and are expressed in terms of Mathieu functions.

In the present work gain is considered as an important parameter for the design of single element antenna and is further enhanced by using antenna array of proper configuration keeping circular polarization intact.

In this paper, a design of circularly polarized $2 \times 2$ planar antenna array of elliptical patches at $2.8 \mathrm{GHz}$ is presented. The patches are excited uniformly. A suitable feed network is designed for impedance matching between the $50 \Omega$ probe and the patches. The design is optimized through the 3D EM simulator HFSS from ANSYS and the prototype is experimentally verified. The paper is organized as follows: Section II describes single element antenna and its performance matrices; Section III deals with the design of $2 \times 2$ antenna array along with the feed network. The design has been experimentally verified in Section IV. Finally, it has been concluded in Section V.

\section{SINGLE PATCH ELEMENT}

\section{A. Design of elliptical microstrip patch element}

To design an antenna array, at first it is required to design a single elliptical patch element and characterize it. Roger's RO4725 substrate having permittivity 2.55 and thickness $1.542 \mathrm{~mm}$ has been used for the present design. The dimension of elliptical patch antenna at the desired frequency of operation is derived from the circular patch. Radius of the circular patch for the fundamental $T M_{110}$ mode is given by [8]

$$
\begin{aligned}
& \mathrm{a}=\frac{F}{\left(1+\frac{2 h}{\pi F a_{r}}\left[\ln \left(\frac{\pi F}{2 h}\right)+1.7726\right]\right]^{1 / 2}} ; \\
& \text { where } \mathrm{F}=\frac{8.791 \times 10^{q}}{F_{F} \sqrt{\varepsilon_{F}}}
\end{aligned}
$$


$h=$ height of the substrate,

$\varepsilon_{\mathrm{r}}=$ permittivity of the substrate and

$f_{\mathrm{r}}=$ desired resonant frequency.

Radius of the circular patch is calculated to be $18.4 \mathrm{~mm}$ for the present substrate at $2.8 \mathrm{GHz}$. It is taken as the minor axis of the ellipse and the major axis is taken as $18.8 \mathrm{~mm}$ to perturb the circular patch for the excitation of even and odd modes along the two perpendicular axes.

\section{B. Simulation Results}

The single elliptical patch element is shown in Fig. 1. Probe feeding technique is used to excite the elliptical microstrip patch antenna where feed is located at a radial distance 10 $\mathrm{mm}$ from the centre of the ellipse at an angle $45^{\circ}$ with respect to the major axis. The 3D EM simulator HFSS from ANSYS is used for the simulations with an appropriate size of radiation boundary. Simulated $S$-parameters are shown in Fig. 2. It ensures that the patch is resonating at $2.8 \mathrm{GHz}$. Fig. 3 shows that the maximum radiation is in the broadside direction and the broadside gain is $3.39 \mathrm{dBi}$. It can be seen from Fig. 4 that CP is also obtained in the broadside direction. The corresponding axial ratio is $2.01 \mathrm{~dB}$.

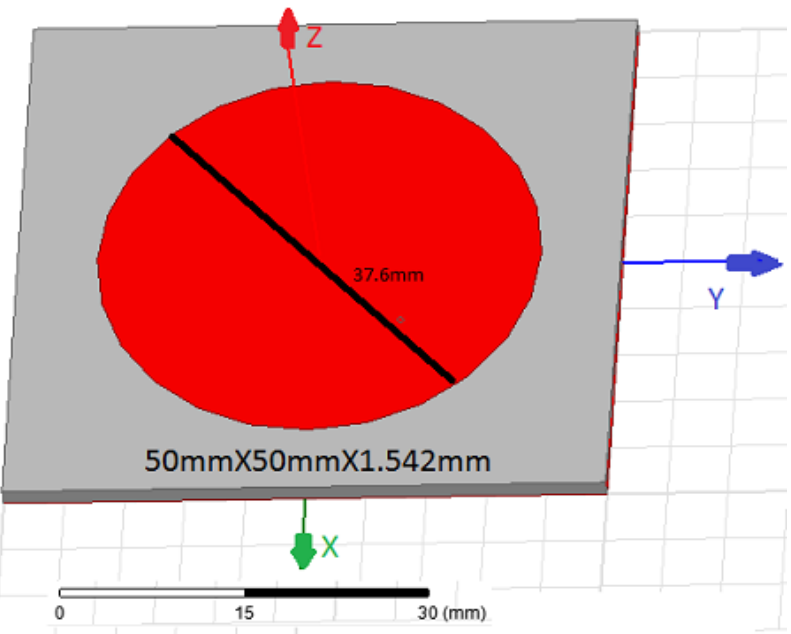

Fig.1 Single element elliptical patch antenna.

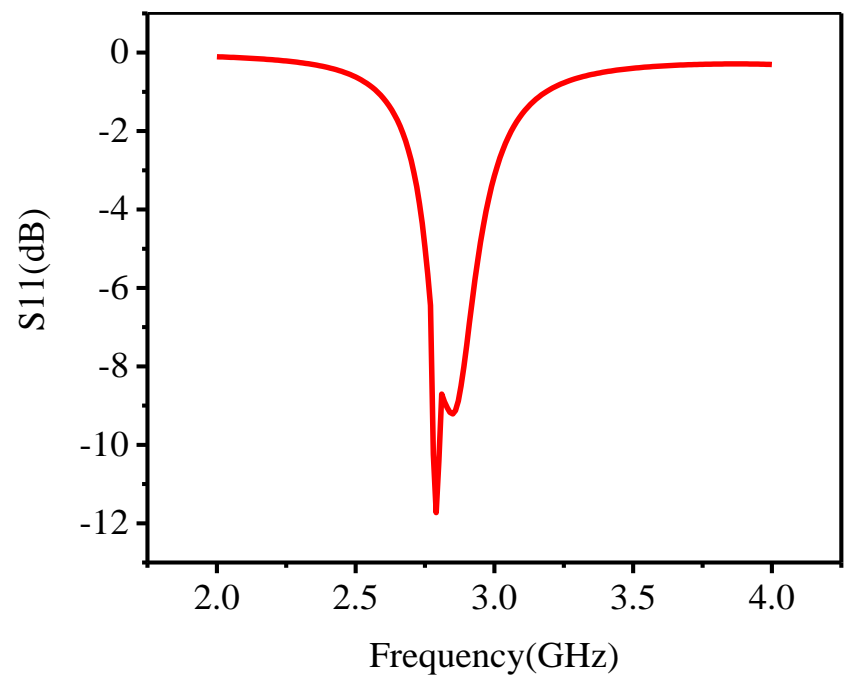

Fig.2 Simulated S11 of the single patch element

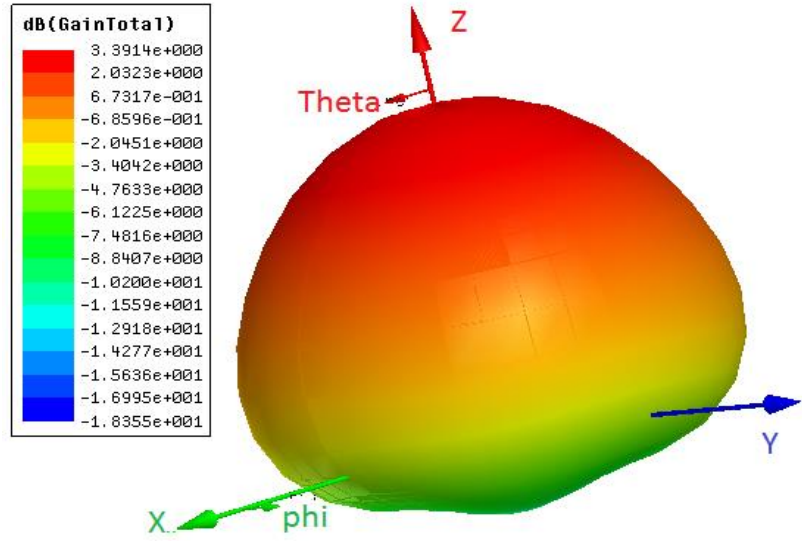

Fig. 3 Simulated 3D radiation pattern of the single element

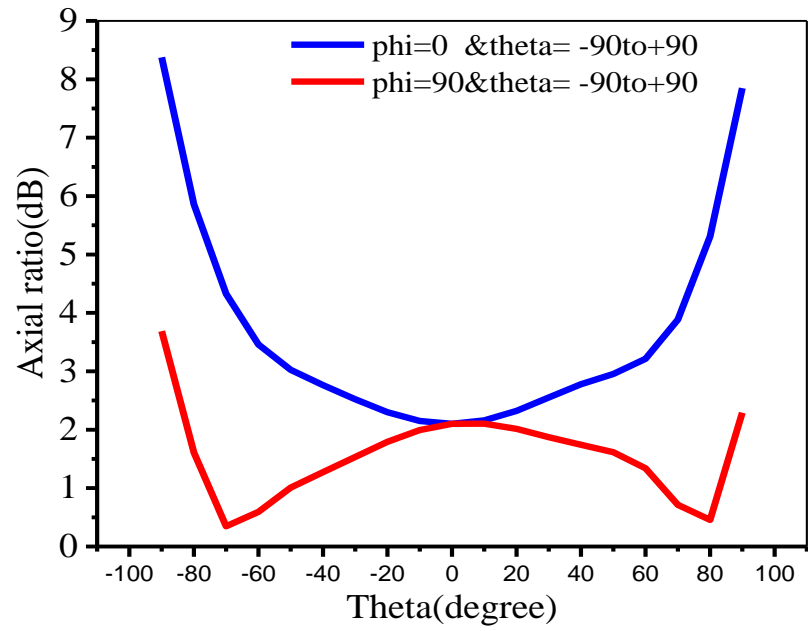

Fig. 4 Simulated axial ratio for the single element.

\section{DESIGN OF AN ANTENNA ARRAY}

\section{A. Theory}

Antenna array can be formed by assembling the radiating elements in geometrical and electrical configuration without increasing the size of the individual elements to achieve high gain. The total field radiated by the antenna array will be vector addition of radiated fields of individual elements. The array factor defined for $\mathrm{M} \times \mathrm{N}$ elements while the individual elements are excited uniformly is given as [2]

$$
\begin{aligned}
& \left(A F_{n}\right)(\theta, \emptyset)=\left\{\frac{1}{M} \frac{\sin \left[\frac{M}{2} \varphi_{x}\right)}{\sin \left(\frac{1}{2} \varphi_{x}\right)}\right\}\left\{\frac{1}{N} \frac{\sin \left(\frac{\omega}{2} \varphi_{y}\right)}{\sin \left(\frac{1}{2} \varphi_{y}\right)}\right\} \\
& \text { where, } \varphi_{x}=\mathrm{k} d_{x} \sin \theta \cos \emptyset+\beta_{x} \\
& \varphi_{y}=k d_{y} \sin \theta \sin \emptyset+\beta_{y} .
\end{aligned}
$$

Proposed array structure of size $2 \times 2$, i.e., $M=2 ; N=2$ is designed by considering the patches of elliptical shape.

\section{B. $2 x 2$ array design}

The elliptical patch element designed in Section 2 is used for the array design. To avoid mutual coupling between antenna array elements, inter-element spacing is chosen as $\lambda_{\mathrm{g}}$. The corporate feed structure is considered to excite the individual antenna element as shown in Fig. 5. The feedline is connected at the edge of individual patch at angle of $45^{0}$ to excite the odd and even $T M_{110}$ modes necessary for circular polarization. 
Input impedance of each single patch is the minimum at the centre of the patch and the maximum at the edge of the patch. The antenna input impedance at the edge is found to be $100 \Omega$ with a small imaginary part. So, all of the elliptical patches are connected to $100 \Omega$ lines each. Hence, the equivalent impedance at the junctions of each pair is $50 \Omega$. A quarter-wave impedance transformer at $2.8 \mathrm{GHz}$ is placed between a $50 \Omega$ impedance and $100 \Omega$ line whose characteristic impedance is calculated as $\mathrm{Z}=\sqrt{Z_{\text {in }} \times Z_{\text {out }}}=$ $\sqrt{50 \times 100} \Omega=70.7 \Omega$. Finally, the two pairs of $100 \Omega$ lines from each side results in an equivalent of $50 \Omega$ where the co-axial feeding is considered. The simulated 3D radiation pattern of the antenna array is shown in Fig. 6. There is no side lobe. Gain in the broadside direction is found to be 9.82 $\mathrm{dBi}$. Fig. 7 shows that the $3 \mathrm{~dB}$ axial ratio bandwidth is 15.4 $\mathrm{MHz}$ around $2.8 \mathrm{GHz}$.

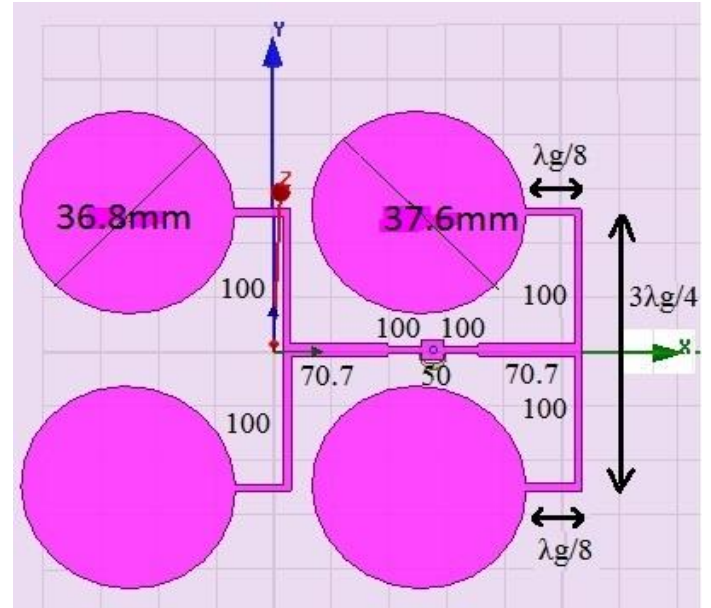

Fig. 5. Configuration of the $2 \times 2$ elliptical patch antenna array.

The substrate size is $150 \times 150 \times 1.542$. Semi major axis of each individual patch $=18.8$ and semi minor axis $=18.4$. Total length of $100 \Omega$ line $=\lambda_{2} @ 2.8 \mathrm{GHz}=67.30$, width of the $100 \Omega$ line $=1.20$, length $70.7 \Omega$ line $=\frac{z_{2}}{4}=16.83$, width of $70.7 \Omega$ feedline $=2.36$, width of $50 \Omega$ feedline $=3.80$. All dimensions are in $\mathrm{mm}$.
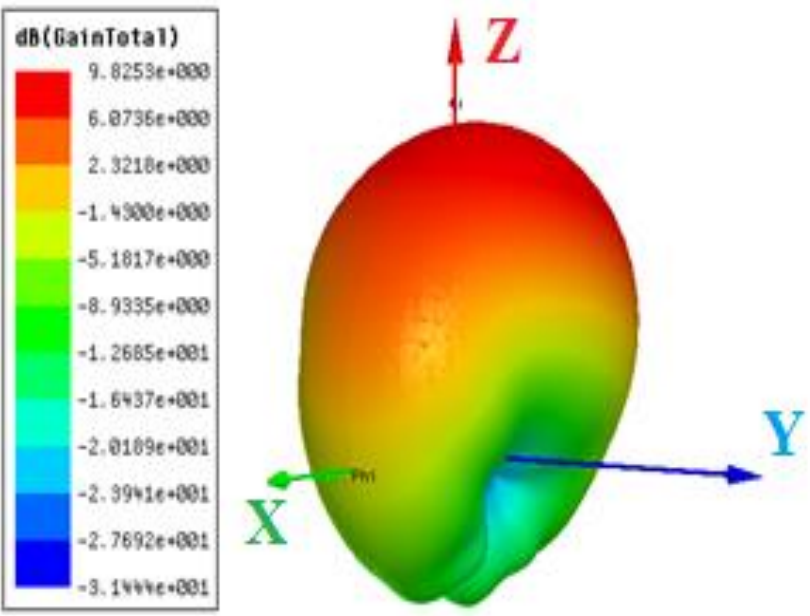

Fig.6. Simulated 3D Radiation Pattern of the antenna array.

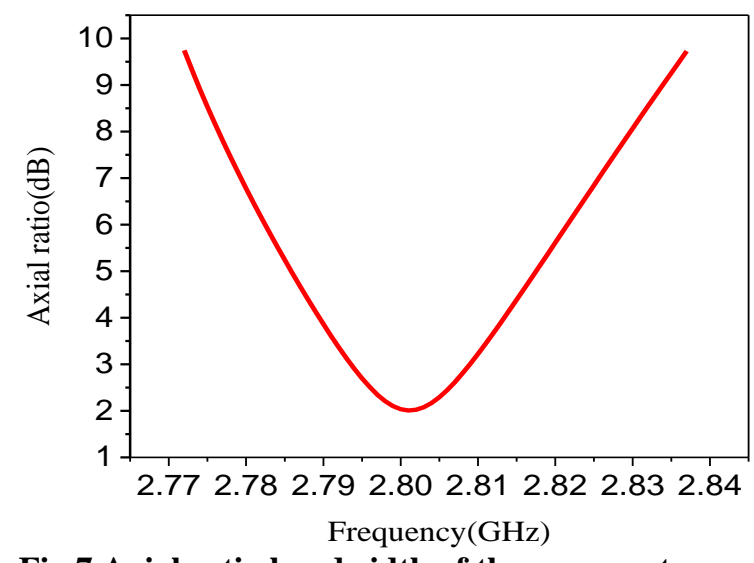

Fig.7 Axial ratio bandwidth of the array antenna.

\section{FABRICATION AND MEASUREMENT RESULTS}

The designed array antenna has been fabricated using the same substrate. A photograph of the fabricated antenna is shown in Fig. 8. Measured and simulated $S_{11}$ of the designed prototype is shown in Fig. 9 shows good impedance matching. Radiation pattern of the antenna has been measured in laboratory environment and the normalized radiation patterns at the two principal planes are shown in Fig. 10. Measured axial ratio in the broadside direction is obtained as $1.93 \mathrm{~dB}$. The axial ratio measurement set up is shown in Fig. 11.

Gain of the designed antenna array has been measured using a horn antenna with known gain as the transmitting antenna. Friis Transmission Law for the received power calculation is given by [2]

$$
P_{y}=G_{t} G_{y} P_{t}\left(\frac{\lambda}{4 \pi r}\right)^{2}
$$

where, $P_{\mathrm{t}}=$ Transmitted power,

$P_{\mathrm{r}}=$ Received power,

$G_{\mathrm{t}}=$ Gain of the transmitting antenna,

$G_{\mathrm{r}}=$ Gain of the receiving antenna,

$r=$ Distance between the transmitting and receiving antennas and $\lambda=$ Wavelength corresponding to operating frequency.

Considering $P_{\mathrm{t}}=-15.1 \mathrm{dBm}, P_{\mathrm{r}}$ is obtained as $-42.1 \mathrm{dBm}$ at $2.8 \mathrm{GHz}$ at a distance of $132 \mathrm{~cm}$. Hence, the gain of the fabricated antenna array is found to be $8.2 \mathrm{dBi}$ while the simulated realized gain of the designed prototype is $9.82 \mathrm{dBi}$

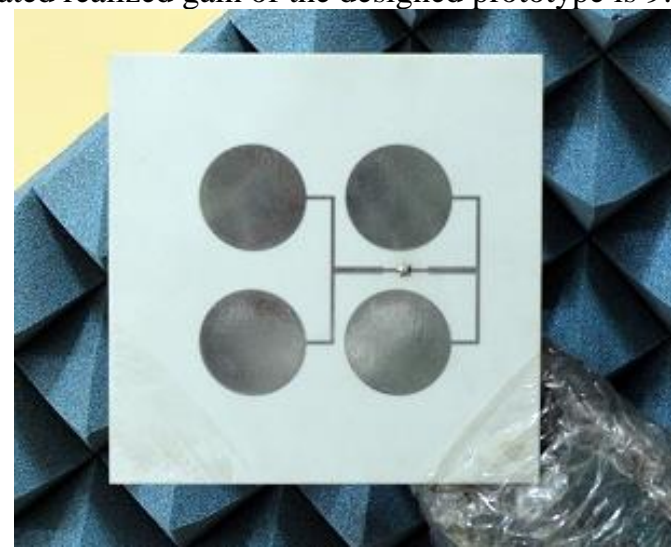

Fig. 8 Photograph of the fabricated antenna array. 


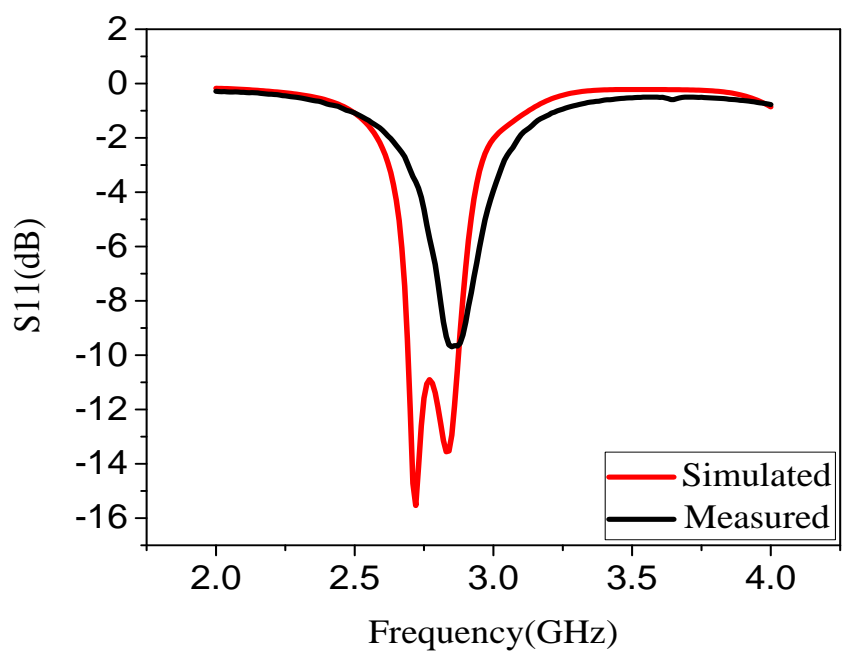

Fig. 9 Measured and simulated $S_{11}$

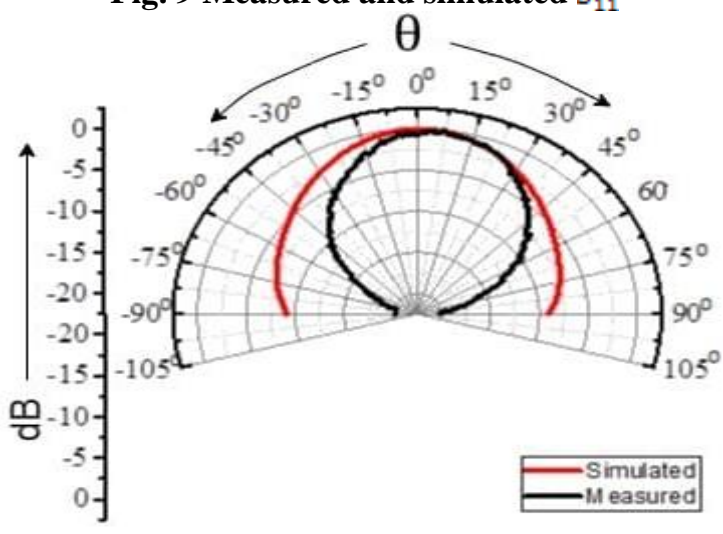

(a)

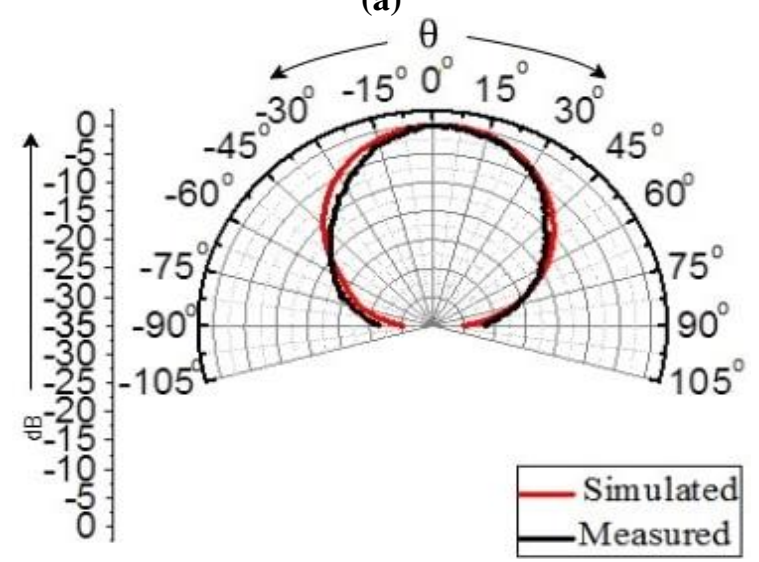

(b)

Fig.10 Measured and simulated normalized radiation patterns at the plane (a) $X-Z$ and (b) $Y-Z$.

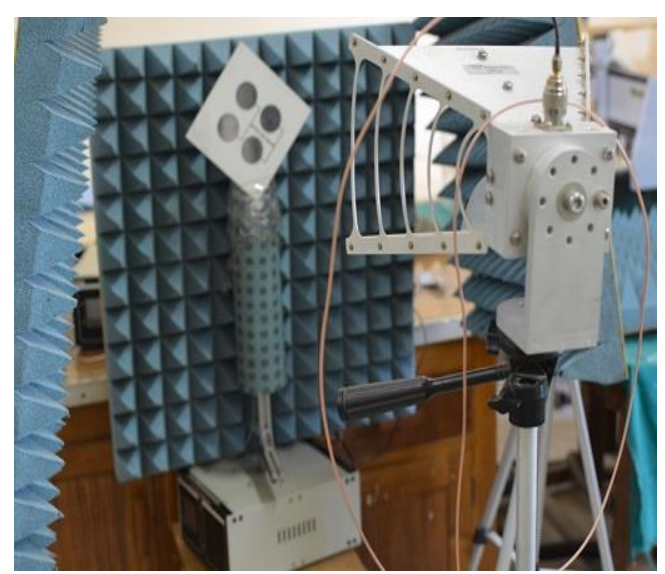

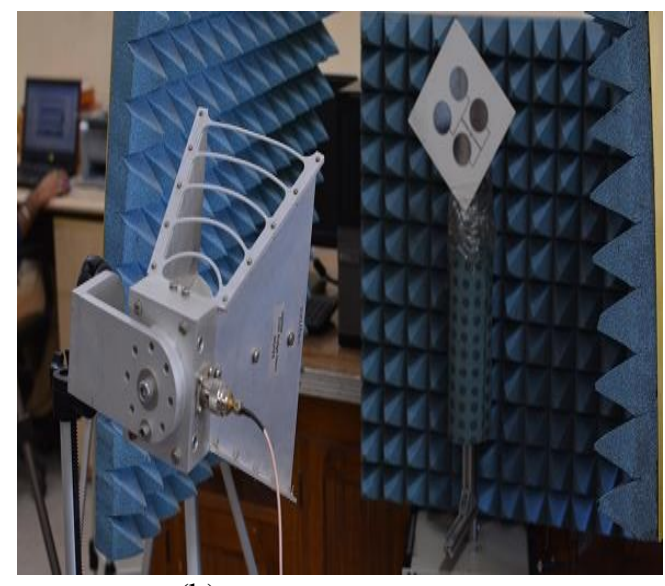

(b)

Fig. 11 Axial ratio measurement set up.

\section{CONCLUSION}

Detailed design procedure, fabrication and measurements of a circularly polarized antenna array of elliptical patches have been demonstrated. Experimental results show good agreement with measurements. Primary advantages of the presented antenna array are: good circular polarization characteristics, single feed, structural simplicity, and no lobes. Here, a design example of a planar antenna of $2 \times 2$ patches at $2.8 \mathrm{GHz}$ has been reported. Similar design procedure can be extended for more number of array elements at the desired frequency of operation and can find applications for Global Positioning System, Global Navigation Satellite System and other communication systems where circular polarization is required.

\section{REFERENCES}

1. K. R. Carver and J. W. Mink, "Microstrip antenna technology," IEEE Trans. Antennas Propagat., vol. AP-29, no. 2, pp. 2-24, Jan. 1981.

2. C.A. Balanis Antenna Theory, Analysis and Design, John Wiley \& Sons India, 2005.

3. G. L. Lan and D. L. Sengupta, "Post-tuned single feed circularly polarized patch antenna," in IEEE. AP-S Int. Symp. Digest, pp. 85-88, Jun. 1985.

4. H. D. Weinschel. "A cylindrical array of circularly polarized microstrip antennas, in IEEE AP-S Int. Symp. Digest, pp. 175-180, Jun. 1975.

5. H. Iwasaki, "A circularly polarized small-size microstrip antenna with a cross slot," IEEE Trans. Antennas Propagat., vol. 44, no. 10, pp. 1399-1401, Oct. 1996.

6. I. P. Yu, "Low profile circularly polarized antenna," NASA Rep. N78-15332, 1978.

7. L.C. Shen, "The elliptical Microstrip Antenna with Circular Polarization," IEEE Trans. Antennas Propagat.,vol.AP-29, No.1, Jan 1981.

8. I. J. Bahl and P. Bhartia, Microstrip Antenna. Dedham, MA: Artech House, 1980.

(a)

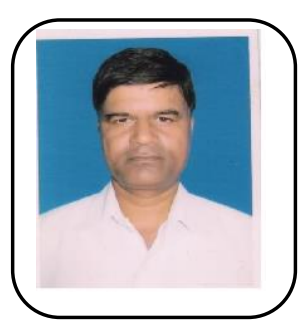

Life member of ISTE.

\section{AUTHOR'S PROFILE}

Rabindra Kumar was born in Bihar,India in 1957. He received the Bachelor's degree in Electrical Engg in MIT Muzaffarpur, Bihar University in 1985 and M.Tech from I.T BHU now IIT BHU Varanasi in 1987. He joined Bihar College of Engg Patna now NIT Patna as faculty member in 1987 and continuing till date. His field of interest are Antenna and Microwave Engg. He is a member of IEEE, Fellow member of IETE and

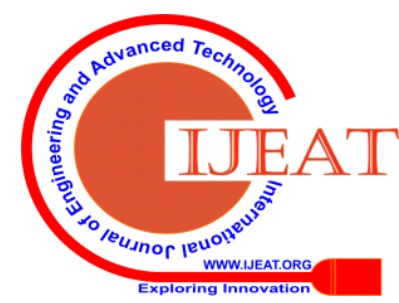




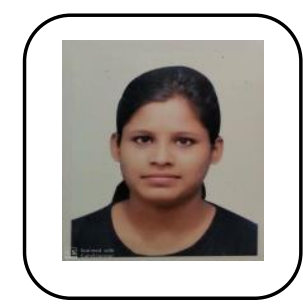

Neha kumara was born in Dhanbad India in 1994. She received the Bachelor's degree in Electronics and Communication Engg from JIS College of Engg, MAKAUT University of Kolkata in 2017 and pursuing M.Tech from NIT Patna. Her field of interest is Microstrip Patch Antennas.

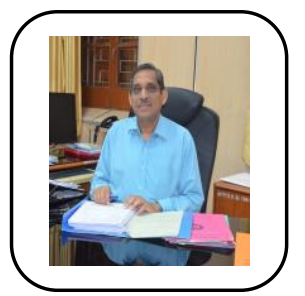

Pradeep Kumar Jain was born in Varanasi, India in 1958. He received Bachelor's degree in Electronics Engg in 1979, Master degree in Microwave Engg in 1981 and Ph.D degree in 1988 all from IT BHU now IIT BHU Varanasi. He joined IT BHU Varanasi as faculty in 1981. He joined as Director at NIT Patna in Nov 2017 and continuing till date. His research fields are High Power RF/Microwave Devices Circuits and Systems, RF MEMs, Meta material devices, Microwave Imaging and Remote Sensing. He is a senior member of IEEE , Fellow member of IETE, Fellow member of Institution of Engineers of India, Fellow member of VEDA Society. He has published more than hundred papers in International Journals.

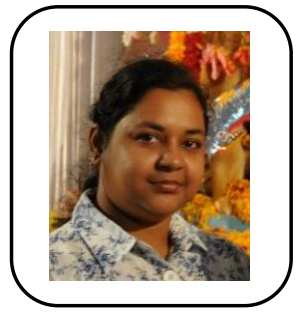

Priyanka Mondal was born in Calcutta in 1976. She received Bachelor's degree in Electronics Engg in 2002 and Master degree in Electronics Engg in 2004 both from Institute of Radio Physics and Electronics, Kolkata Calcutta University. She joined NIT Patna in 2015 as faculty member and continuing. Her research field are Microwave Millimeter wave components for wireless communication systems. She is a senior member of IEEE. 